\title{
Anabases
}

ANABASES Traditions et réceptions de l'Antiquité

$23 \mid 2016$

Varia

\section{Antiquités parallèles (4). Grèce antique : en tics ou en toc?}

Claude Aziza

\section{(2) OpenEdition}

Journals

Édition électronique

URL : http://journals.openedition.org/anabases/5639

DOI : 10.4000/anabases.5639

ISSN : 2256-9421

Éditeur

E.R.A.S.M.E.

\section{Édition imprimée}

Date de publication : 2 mai 2016

Pagination : 157-161

ISSN : 1774-4296

\section{Référence électronique}

Claude Aziza, «Antiquités parallèles (4). Grèce antique : en tics ou en toc ? », Anabases [En ligne], 23।

2016, mis en ligne le 02 mai 2019, consulté le 20 octobre 2019. URL : http://journals.openedition.org/ anabases/5639; DOI : 10.4000/anabases.5639 
Anabases 23 (2016), p. I57-16

\section{Antiquités parallèles (4) Grèce antique: en tics ou en toc?}

Claude AzızA

a tarte à la crème du miracle grec qu’ont dégustée, bouche bée et les yeux fermés, des générations d'hellénistes n’a guère nourri la pellicule. Sur environ deux milliers de films “à l'antique», on en trouve moins d'une cinquantaine sur la Grèce historique. À croire que celle-ci ne comporte aucun des éléments propres à captiver un public essentiellement populaire. Rome flatte le goût de l'outrance, la Bible, celui du sacré. L'Égypte a son mystère, la mythologie, son merveilleux. Ce sont là les points cardinaux d'un cinéma qui cherche d'abord à faire rêver, rarement à faire comprendre.

Certes la Grèce n'est pas avare en mirages : celui de la Cité, où règnent la Démocratie, la Justice, le respect des Lois, par exemple. Mais on n'exige pas à l'entrée des salles de cinéma des spectateurs-géomètres! Bref, avouons-le, la Grèce historique, pas celle des légendes s'entend, manque de goût et le monde attique de sel. Quant au brouet spartiate...

Rome a une histoire, la Grèce en a plusieurs. On y chercherait en vain, sinon des héros, du moins des monstres sacrés et les Vies parallèles du bon Plutarque peinent à trouver, face au Romain, le Grec de même pointure. Que valent donc un Périclès ou un Thémistocle face à un César ou un Néron? Où trouver une Cléopâtre? Où une Messaline? Faute de Poppée il faut faire avec Aspasie.

"C'est une gamine charmante, charmante, charmante / Qui possède une âme innocente, innocente / En elle tout est poésie / Elle répond au joli nom d'Aspasie. » Ainsi chantait Phi-Phi, dans l'opérette homonyme d'Henri Christiné (Igi8), accommodant à la sauce Châtelet l'Athènes de Phidias. Le cinéma y trouva prétexte, en I927, à une adaptation. Célébrant, comme il convient, et à sa façon, les quelques beautés hellènes pourvues d'un embryon d'historicité. Sapho de Lesbos, bien sûr, auréolée du soufre des amours interdites. Mise en scène dès Igog par l'Italien 
Novelli, elle réapparaît de-ci, de-là : en ıgog, I9ı2, I9ı8. Pietro Francisci, sans faillir à la légende, la fait rentrer dans le rang: dans Sapho, Vénus de Lesbos (I96i), elle n'aime que le beau Faon...

Le refus de l'homme semble bien être le seul fil qui relie Sapho à Lysistrata. Présente sur les écrans depuis ıgı, par Louis Feuillade, elle fut incarnée avec grâce par Martine Carol, dans un des sketches, dû à Christian-Jaque, de Destinées, en cette année I954 qui vit aussi réapparaître à l'écran, depuis Igoo, la courtisane Phryné (Phryné, courtisane de l'Orient, par Mario Bonnard) sans voiles et tous charmes dehors. Fin des femmes grecques, si l'on ajoute, par pur souci d'exhaustivité, les Jeunes Aphrodites, de Nicos Koundouros (I962).

Et du côté des hommes? La moisson est tout aussi maigre: les deux amis libérateurs de Syracuse: Damon et Pythias (I9o8, I9o9, ı9ı par Louis Feuillade, I9I4, I955, I963); Socrate, au seuil du trépas (I9o9, I940, I953, I966, I970 à la télé par Roberto Rossellini, I97I). Dans l'adaptation du Banquet de Platon par Marco Ferreri (I988), il est incarné par Philippe Léotard. Il est aux prises avec Xanthippe, son acariâtre épouse dans un téléfilm de ig53. La mort d'Empédocle a été filmée par Jean-Marie Straub et Danielle Huillet (La Mort d'Empédocle, I987), Hippocrate, le fut, en I972, par Dimis Dadiras (Hippocrate et la démocratie). Le fabuliste Ésope, lui, a eu droit à cinq films (1946, г953, I957, I968, г97I).

\section{Les guerres médiques}

Un seul conflit, en définitive, a donné naissance à quatre superproductions : les guerres médiques. Dans les années ig6o, deux Américains en illustrèrent, chacun à sa façon, deux temps forts. Rudolph Maté, dans La Bataille des Thermopyles (I96I), montra un Léonidas haut en couleurs dans un chromo d'Épinal. Jacques Tourneur, lui, en I959, avait bénéficié de l'aide du talentueux Mario Bava pour une Bataille de Marathon, avec un improbable Philippidès (le coureur de), une blondinette nunuche et une brune brûlante.

Un demi-siècle plus tard, le conflit s'est musclé. Dans 3oo, un film de Zack Snyder (2007), d'après une BD de Frank Miller (2004), Léonidas rappelait par sa plastique que son nom était désormais associé à des tablettes de chocolat. Quant à Xerxès, sa transformation en drag-queen excita l'ire de l'Iran, délirant sur sa lyre nationaliste. Le pastiche ajouta un brin de fantaisie fort gai et gay, un an plus tard, avec Spartatouille (J. Friedberg et A. Seltzer, 20o8).

Sous le même label est sortie, en 2014, une bataille de Salamine (N.Murro). Dont l'essentiel se résume à une angoissante question. Est-ce un spin-off qui gloserait sur un personnage secondaire de 300 ? Est-ce une séquelle, qu'autoriserait la chronologie? Et si c'était une préquelle, où seraient mis en place, en amont, des éléments importants pour la compréhension du film? Dont le héros est ici Thémistocle face à la reine Artémise Ire (Eva Green), mi-diablesse, mi-amazone, dont le 
bustier-cuirasse évoque vaguement le buste du Léonidas de 300 et dont le passage “à la force obscure perse » (sic) rappelle que le Vador (Dark) est toujours debout.

Mais c'est dans l'ambition explicitement avouée du réalisateur, un quasiinconnu, que réside - peut-être - l'intérêt du film. Il s'était promis - avec la spectaculaire bataille navale - d'égaler, au xxi ${ }^{\mathrm{e}}$ siècle, Ben-Hur et Cléopâtre réunis. Ambition à moitié réussie, trop souvent contrariée par les tics inhérents à un genre si manichéen et le toc de reconstitutions, fondées trop souvent sur le spectaculaire plutôt que sur l'exactitude historique.

\section{Alexandre Le Grand}

Reste le seul héros qui put rivaliser avec ceux de la mythologie, de la Bible et de Rome réunies, Alexandre le Grand. Bizarrement, la figure du conquérant, si souvent illustrée dans le cinéma indien, n’a guère inspiré les cinéastes occidentaux. S'il apparaît en filigrane dans L'Homme qui voulut être roi (John Huston, I975) et dans DrFaustus (Richard Burton, I967), il a été incarné - avec grandeur, il est vrai - à l'écran, justement par ce même Burton, dans un beau film de Robert Rossen (Alexandre le Grand, I956) qui se veut plus une méditation sur le pouvoir qu'une épopée spectaculaire. Le film d'Oliver Stone qui suivit en 2005 ne remporta pas l'unanimité par son côté un peu trop manichéen.

Aphrodite réapparaît aussi au moment du conflit entre la Grèce et la Macédoine, dans une histoire que nous content deux vieux routiers du mélo à l'antique, Fernando Cerchio et Vladimir Tourjansky. Dans Aphrodite, déesse de l'amour (1957), il s'agit d'une humble mortelle, devant le corps de laquelle des générations ont rêvé, depuis plus de deux mille ans : celle qui fut le modèle de Praxitèle, l'Aphrodite de Cnide, le premier nu intégral, semble-t-il, de la statuaire antique. Et dont on ne pourrait avoir qu'une bien pâle idée avec l'Aphrodite d'Arles. Le modèle, on s'en doute, rendra fou l'artiste, nouveau Pygmalion. Mais l'ingrate jeune fille préférera, au bout d'un chemin semé d'embûches, la virile étreinte d'un beau Macédonien.

On retrouvera la Grèce hellénistique dans La Révolte de Sparte (Alberto de Martino, I964) et dans La Bataille de Corinthe (Mario Costa, I96I).

\section{Bilitis}

Le I2 décembre ı894 paraissent Les Chansons de Bilitis traduites du grec pour la première fois par P.L. Contemporaine de la poétesse Sapho et originaire de Pamphylie, Bilitis dont la vie occupe les premières pages du recueil, a vécu ensuite à Lesbos, pour mourir à Chypre. Ses poésies pleines de grâce et de fraîcheur, étaient gravées sur le tombeau de la poétesse, découvert à Chypre peu de temps auparavant par l'archéologue allemand Heim, qui en fit une fort savante édition. 
C'est cette édition que traduit, à l'exception de sept chansons, P.L., alias Pierre Louÿs, déjà traducteur des Grecs Méléagre et Lucien.

La critique s'enthousiasma pour le recueil. Les hellénistes et les philologues aussi ; l'un des plus éminents d'entre eux, Gustave Fougère, professeur d'archéologie grecque à l'université de Lille et ancien élève de l'École d'Athènes, proposa fort aimablement à l'auteur de lui donner "quelques variantes de traduction » à partir de textes originaux. Un autre savant n'hésite pas à lui écrire: “Bilitis et Méléagre ne sont pas pour moi des inconnus, mais ce seront désormais des amis personnels. „ Deux ans plus tard, Jean Bertheroy, romancière qui écrira en I9o5 La Danseuse de Pompéi, présente, dans la Revue des jeunes filles, une traduction nouvelle, en vers, de six chansons. Elle avait, disait-elle, travaillé directement sur l'édition Heim. En ıوı, dans sa Bibliographie pratique de la littérature grecque, Paul Masqueray signale les poèmes de Bilitis, en notant cependant la médiocrité de la traduction de Pierre Louÿs.

Bel exemple de la suffisance alliée à l'ignorance! Quelle leçon de rigueur scientifique! Quel exemple d'honnêteté intellectuelle! Car Bilitis n'a jamais existé. Ni, bien sûr, d'archéologue nommé Heim. Son modèle ne fut qu'une jeune et charmante Ouled-Naïl, Meryem Bent Ali, auprès de laquelle, à Constantine, Pierre Louÿs trouva l'inspiration. Superbe mystification littéraire due à un faussaire de génie. Mais quand l'inspiration - comme dans la Bilitis de David Hamilton (I976), tirée de l'œuvre de l'écrivain - s'arrête aux jeux de mains de jeunes filles en bouton, sous emballage mode avec caméra évanescente et luminosité pastel, il ne reste du diamant d'origine que le tape-à-l'œil du zircon.

\section{Un colosse aux pieds d'argile}

Sergio Leone, réalisateur du Colosse de Rhodes, on le sait peu, a été nourri du lait de la louve romaine et élevé dans l'univers du péplum. Quand il naît en I929, son père Roberto Roberti (un pseudonyme pour Vincenzo Leone) a, entre autres films, tourné un Maciste, et son parrain, Mario Camerini, un autre.

Le jeune Sergio fréquente assidûment les studios, il devient l'assistant des grands du péplum : Carmine Gallone (Scipion l'Africain, I937, Carthage en flammes, I959) et Alessandro Blasetti, dont la Fabiola, en i948, sonne le retour de l'Italie et du péplum sur les écrans. Quoi d'étonnant alors si Leone participe aux superproductions américaines qui s'installent - économies obligent - à Cinecittà? Il s'occupe des figurants de Quo Vadis? en ig5r; assiste Raoul Walsh pour la seconde équipe d'Hélène de Troie, de Robert Wise, en I956; tourne enfin, auprès d'un Andrew Marton défaillant, la course de chars de Ben-Hur, en ig59.

Entre-temps, il a fait des scénarios ou des découpages pour Sous le signe de Rome, du vétéran Guido Brignone (I959), Romulus et Remus dont il laissera, en I96I, la mise en scène à Sergio Corbucci, Phryné, courtisane d'Orient (ı553), de Mario 
Bonnard. C'est ce même Bonnard qu'il assiste pour les Derniers Jours de Pompéi, en I959, et qu'il remplace pour cause de maladie. Le succès du film incite les producteurs à lui confier sa première mise en scène, en ig6o, celle du Colosse de Rhodes.

C'est là que Leone va régler ses comptes - il le dit implicitement - avec le péplum. Peut-être aussi avec son enfance, sa famille, son pays. Car si, dans $L e$ Colosse de Rhodes, il y a tous les ingrédients du genre, ils sont parodiés, détournés, démontés. Le titre lui-même annonce la couleur : loin de renvoyer à un bel athlète, style Hercule ou Maciste, il fait allusion à la gigantesque statue (35 mètres) que les Rhodiens, après le siège de Démétrios Poliorcète (3o5-3o4 avant J.-C.), durent construire en hommage à Apollon. On ne sait exactement où fut érigée cette statue de bronze - mais sans doute pas devant le port - qui fut détruite par un tremblement de terre en 227 avant J.-C., et dont les ruines perdurèrent à même le sol pendant presque un millénaire.

Mais Leone n'a que faire de l'Histoire. Il raconte les vacances d'un jeune et beau général athénien, Darios, impliqué malgré lui dans un triple complot: une révolte des citoyens, une coalition entre Rhodes et les Phéniciens (peu importe s'ils ne jouent plus à l'époque aucun rôle) contre les Grecs, une intrigue de palais entre le chef du gouvernement et son premier ministre félon... Darios, antihéros, nonchalant et cynique, finira par s'animer après tortures, aventures avec héroïnes contrastées et combats dans les entrailles du Colosse véritable merveille de la technique antique. Au tremblement final s'ajoutera, pour bien faire, un raz-demarée. Histoire de dire que le péplum - colosse aux pieds d'argile - est bien mort, d'indigestion cinématographique. Et lorsque Leone aura aussi déchiré à belles dents la matière de l'Ouest, il pourra alors être enfin lui-même, loin des ombres de Roberto Roberti et de John Ford.

\section{Claude Aziza}

Université de la Sorbonne Nouvelle, Paris III claude.aziza@laposte.net 